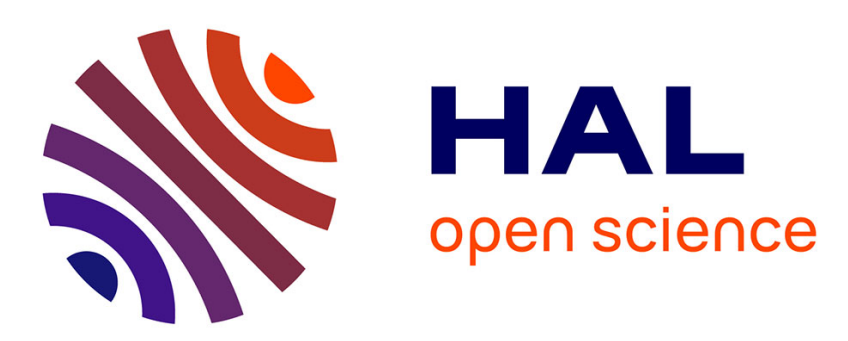

\title{
Mesure de l'énergie des ions lourds par la méthode des protons projetés
}

\author{
R. Bimbot, S. Della Negra, D. Gardes, H. Gauvin, B. Tamain
}

\section{To cite this version:}

R. Bimbot, S. Della Negra, D. Gardes, H. Gauvin, B. Tamain. Mesure de l'énergie des ions lourds par la méthode des protons projetés. Revue de Physique Appliquée, 1978, 13 (8), pp.393-398. 10.1051/rphysap:01978001308039300 . jpa-00244466

\section{HAL Id: jpa-00244466 https://hal.science/jpa-00244466}

Submitted on 1 Jan 1978

HAL is a multi-disciplinary open access archive for the deposit and dissemination of scientific research documents, whether they are published or not. The documents may come from teaching and research institutions in France or abroad, or from public or private research centers.
L'archive ouverte pluridisciplinaire HAL, est destinée au dépôt et à la diffusion de documents scientifiques de niveau recherche, publiés ou non, émanant des établissements d'enseignement et de recherche français ou étrangers, des laboratoires publics ou privés. 
Classification

Physics Abstracts

$06.90-25.70-29.90$

\title{
MESURE DE L'ÉNERGIE DES IONS LOURDS PAR LA MÉTHODE DES PROTONS PROJETÉS
}

\author{
R. BIMBOT, S. DELLA NEGRA, D. GARDÈS, H. GAUVIN et B. TAMAIN \\ Laboratoire de Chimie Nucléaire, Institut de Physique Nucléaire, B.P. nº 1, 91406 Orsay, France
}

(Reçu le 16 mars 1978, accepté le 8 mai 1978)

\begin{abstract}
Résumé. - Un dispositif destiné à la mesure de l'énergie des faisceaux d'ions lourds de 3 à $6 \mathrm{MeV} /$ uma a été construit. Le principe de la méthode est de mesurer l'énergie des protons projetés à zéro degré par collision élastique des ions incidents avec les noyaux d'hydrogène d'une cible de formvar. L'incertitude calculée sur l'énergie ainsi mesurée pour les ions lourds est de $\pm 0,45 \%$. Des mesures faites sur des faisceaux de ${ }^{19} \mathrm{~F}$ et ${ }^{40} \mathrm{Ca}$ d'énergie bien connue, accélérés par un Tandem MP, ont montré un écart maximum de $0,3 \%$ entre les énergies réelles et mesurées. Le dispositif permet de contrôler ou calibrer des méthodes plus lourdes de détermination de l'énergie des ions lourds : déviation magnétique, temps de vol. Il se prête particulièrement bien à la mesure des pertes d'énergie d'ions lourds dans des ralentisseurs solides.
\end{abstract}

Abstract. - A device has been built to measure the energies of 3 to $6 \mathrm{MeV} /$ uma heavy ion beams. Schematically the method consists in the energy measurement of the proton knocked forward at $0^{\circ}$ by elastic scattering of heavy ions with the hydrogen nuclei of a thin formvar target. The calculated accuracy of the measured energy of heavy ion is $\pm 0.45 \%$. Measurements were done with ${ }^{19} \mathrm{~F}$ and ${ }^{40} \mathrm{Ca}$ beams from a MP Tandem at well known and precise energies. The maximum deviation between exact and measured energies is $0.3 \%$. This device can be used to control or to calibrate more sophisticate methods of heavy ion energy determinations : magnetic analysis, time of flight. The device is very suitable for measuring heavy ion stopping powers in solid absorbers.

1. Principe de la mesure. - Cette méthode de l'énergie des ions lourds a été indiquée par D. K. Olsen et al. [1]. Son principe est de mesurer l'énergie des protons projetés à zéro degré par collision élastique des ions du faisceau avec une cible de formvar $\left(\mathrm{C}_{5} \mathrm{H}_{10} \mathrm{O}_{2}\right)$. Le détecteur utilisé est une jonction $\mathrm{Si}(\mathrm{Li})$ de 3 à $5 \mathrm{~mm}$ d'épaisseur. Une feuille d'or située entre la cible et le détecteur arrête le faisceau d'ions lourds, mais ne ralentit les protons que de quelques centaines de $\mathrm{keV}$. Enfin, un aimant permanent de 500 gauss (champ moyen) élimine les électrons projetés hors de la feuille d'or. Le schéma du dispositif est donné sur la figure 1 .

1.1 Calculs en mécanique Classique. - 1.1.1 Collision élastique. - Si $E$ est l'énergie du faisceau incident, $E^{\prime}$ son énergie moyenne dans la cible de formvar, l'énergie du proton éjecté à l'angle $\theta$ vaut :

$$
E_{\mathrm{p}}(\theta)=4 M m E^{\prime} \cos ^{2} \theta /(M+m)^{2}
$$

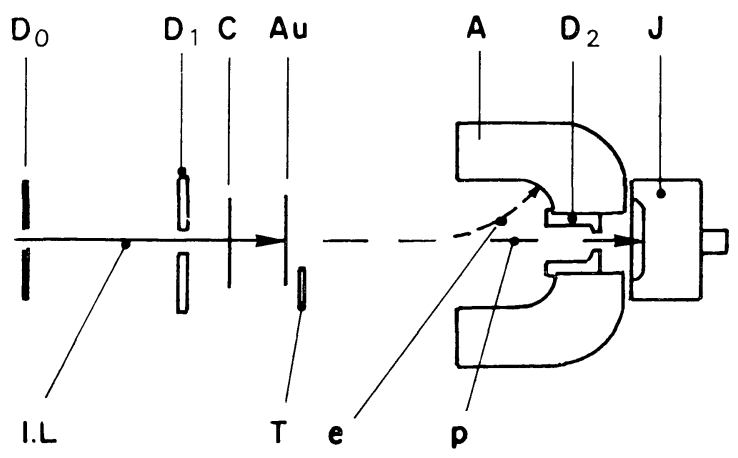

Fig. 1. - Dispositif de mesure de l'énergie des ions lourds (schéma de principe). $\mathrm{D}_{0}, \mathrm{D}_{1}, \mathrm{D}_{2}$ : Diaphragmes; $\mathrm{C}$ : Cible (formvar): $\mathrm{Au}$ : Feuille d'or destinée à arrêter le faisceau d'ions lourds (I.L.) : A : Aimant destiné à dévier les électrons e provenant de la feuille d'or; J : Détecteur à jonction mesurant l'énergie des protons projetés $\mathrm{p} ; \mathrm{T}$ : Dépôt actif de thoron pour calibration.

[Device to measure the energies of heavy ion beams (schematic presentation). $\mathrm{D}_{0}, \mathrm{D}_{1}, \mathrm{D}_{2}$ : collimators; $\mathrm{C}$ : Target (formvar); $\mathrm{Au}$ : Gold foil to stop heavy ion beam ; A : Permanent magnet for elimination of electrons e coming from the gold foil ; J : Detector to measure the energy of knocked protons $\mathrm{p} ; \mathrm{T}: \mathrm{Th} \mathrm{C}$ source for energy calibration.] 
$M$ et $m$ étant les masses de l'ion incident et du proton $\left(^{1}\right)$. Pour $\theta=0^{\circ}$, cette énergie devient

$$
E_{\mathrm{p}}=\left(4 M m /(M+m)^{2}\right) E^{\prime} .
$$

Après traversée de la feuille d'or, les protons ont perdu l'énergie $\Delta E_{\mathrm{p}}$, et l'énergie mesurée dans le détecteur est $E_{\mathrm{p}}^{\prime}=E_{\mathrm{p}}-\Delta E_{\mathrm{p}}$. Par conséquent, l'équation permettant de calculer l'énergie incidente à partir de l'énergie mesurée pour les protons est :

$$
E=\frac{(M+m)^{2}}{4 M m}\left(E_{\mathrm{p}}^{\prime}+\Delta E_{\mathrm{p}}\right)+\Delta E
$$

$\Delta E_{\mathrm{p}}$ est donnée par les relations parcours-énergie des protons dans l'or, connaissant l'énergie $E_{\mathrm{p}}^{\prime}$ et l'épaisseur de la feuille d'arrêt d'or [2].

- $\Delta E$ est la perte d'énergie du faisceau incident dans la demi-épaisseur de la cıble de formvar. Cette perte d'énergie est déterminée en utilisant les pouvoirs d'arrêt tabulés par Northcliffe et Schilling [3], auxquels on applique éventuellement une correction pour tenir compte des récentes déterminations expérimentales [4]. Ces mesures ont montré en effet que les pouvoirs d'arrêt des matériaux légers $\left({ }^{12} \mathrm{C},{ }^{27} \mathrm{Al}\right)$ correctement estimés par les tables pour les ions légers $\left({ }^{16} \mathrm{O}\right)$, sont sous-estimés de $15 \%, 20 \%$ et $30 \%$ respectivement par rapport aux valeurs expérimentales obtenues avec des ions d'argon, de cuivre et de krypton de 4 à $5 \mathrm{MeV} /$ nucléon.

1.1.2 Collision inélastique. - Si la collision entre l'ion lourd et le proton-cible produit l'excitation du noyau incident, l'énergie du proton projeté sera fortement diminuée. L'application des équations de conservation de l'énergie et de la quantité de mouvement, pour $\theta=0^{\circ}$, conduit à l'expression suivante pour l'énergie du proton diffusé inélastiquement dans le système du laboratoire :

$$
E_{\mathrm{pi}} \simeq E_{\mathrm{p}}-\frac{2 M}{M+m} E^{*}-\left(\frac{M}{M+m}\right)^{2} \frac{E^{* 2}}{E_{\mathrm{p}}}
$$

Dans l'équation (4), $E_{\mathrm{p}}$ est l'énergie correspondant à la collision élastique, donnée par l'équation (2) et $E^{*}$ l'énergie du niveau excité. L'écart observé entre les pics correspondant aux collisions élastique et inélastique est donc donné en première approximation par

$$
E_{\mathrm{p}}-E_{\mathrm{pi}} \simeq \frac{2 M}{M+m} E^{*} \# 2 E^{*}
$$

Remarquons dès maintenant qu'en général, ces deux pics seront bien séparés. En effet, pour un faisceau de cuivre d'énergie égale à $265 \mathrm{MeV}$, par exemple,

( ${ }^{1}$ Il est important d'utiliser pour ce calcul les masses réelles et non les nombres de masse. Cette dernière approximation introduirait une erreur de l'ordre de $0,7 \%$, non négligeable par rapport à la précision de la mesure. le calcul donne $E_{\mathrm{p}}=16,44 \mathrm{MeV}$ et, pour le premier niveau excité du cuivre $\left(E^{*}=0,67 \mathrm{MeV}\right)$,

$$
E_{\mathrm{pi}}=15,12 \mathrm{MeV} \text {, }
$$

soit un écart de $1,32 \mathrm{MeV}$, alors que la largeur à mihauteur des pics observée expérimentalement est de l'ordre de 0,250 MeV. Un autre exemple sera donné plus loin.

1.2 Calcul en mécaniQue Relativiste. - L'utilisation des équations de la mécanique relativiste conduit à des résultats différents d'environ 0,1 à $0,3 \%$ des valeurs calculées par la mécanique classique, pour des projectiles d'énergie égale à 3 à $6 \mathrm{MeV}$ par nucléon. Cet écart n'étant pas totalement négligeable par rapport aux incertitudes expérimentales, la mécanique relativiste doit être appliquée chaque fois que l'on désire la précision maximale sur l'énergie mesurée. L'équation permettant de calculer l'énergie du proton diffusé à l'angle $\theta$ est alors

$$
E_{\mathrm{p}}=\frac{2 m B^{2} \cos ^{2} \theta}{1-B^{2} \cos \theta}
$$

avec

$$
B=\frac{\left[E^{\prime 2}-2 M E^{\prime}\right]^{1 / 2}}{M+m+E^{\prime}}
$$

2. Détails expérimentaux. - L'effort de mise au point du système a été principalement effectué dans le but de

- réduire le temps nécessaire à chaque mesure d'énergie (en particulier à la focalisation du faisceau),

- améliorer le rapport signal sur bruit pour les énergies incidentes élevées ( $\geqslant 5 \mathrm{MeV}$ par nucléon),

- obtenir la meilleure précision de mesure.

2.1 Les COllimateurs. - Afin d'assurer autant que possible la condition $\theta=0$, le faisceau était collimaté par le diaphragme $\mathrm{D}_{1}$ et les protons de recul par le diaphragme $D_{2}$. Ces deux diaphragmes avaient un diamètre égal à $3 \mathrm{~mm}$, ce qui, compte tenu de la distance cible- $\mathrm{D}_{2}$ égale à $65 \mathrm{~mm}$, limitait l'ouverture angulaire à $\pm 2^{\circ}$. L'alignement avec la direction du faisceau (définie par l'axe des tubulures) était contrôlé avant l'expérience au moyen d'un laser ou d'une lunette. Dans certaines expériences, un troisième diaphragme $\mathrm{D}_{0}$ a été ajouté, de façon à limiter l'ouverture angulaire due à la convergence du faisceau. Il a été vérifié dans ce cas que le passage à un diamètre de $2 \mathrm{~mm}$ ne modifiait pas l'énergie mesurée.

Pour réduire le bruit produit dans la jonction par les réactions du faisceau sur les collimateurs, leur face avant (regardant le faisceau) a été recouverte d'une feuille d'or de $96 \mathrm{mg} / \mathrm{cm}^{2}$ qui arrête tous les faisceaux utilisés.

2. 2 Les CIBLES DE FORMVAR. - La composition chimique du formvar $\mathrm{C}_{5} \mathrm{H}_{10} \mathrm{O}_{2}$ correspond à un atome-gramme d'hydrogène pour 10,2 $\mathrm{g}$ de cible. Des 
cibles de polystyrène $\left[\mathrm{C}_{6} \mathrm{H}_{5}\left(\mathrm{CH}-\mathrm{CH}_{2}\right)\right]_{n}$ soit $(\mathrm{CH})_{P}$ ont également été utilisées. Elles correspondent à un rendement inférieur de $30 \%$ environ (1 at-g d'hydrogène pour $13 \mathrm{~g}$ ). Le mylar $\mathrm{C}_{10} \mathrm{H}_{8} \mathrm{O}_{4}$, avec 1 at-gH/24 g est à déconseiller.

Les cibles de formvar étaient préparées par la technique usuelle : dépôt sur une plaque de verre par immersion dans une solution contenant environ $6 \mathrm{~g}$ de formvar dans $400 \mathrm{~g}$ de chloroforme et récupération sur un cadre d'aluminium par flottation sur l'eau.

Leur épaisseur était mesurée au point exact d'utilisation par la technique de ralentissement des particules $\alpha$ du dépôt actif du thoron $\left(E_{\alpha}=6,051,6,090\right.$ et $8,785 \mathrm{MeV})$. L'épaisseur optimale adoptée, résultant d'un compromis entre une faible perte d'énergie du faisceau $\Delta E$ et l'obtention d'un nombre suffisant d'événements dans un temps raisonnable, était de l'ordre de $80 \mu \mathrm{g} / \mathrm{cm}^{2}$. L'incertitude sur cette épaisseur était de l'ordre de $\pm 2 \%$.

La tenue des cibles du faisceau était assez bonne pour les ions légers $\left({ }^{16} \mathrm{O},{ }^{19} \mathrm{~F}\right)$ : une cible supportait un faisceau de l'ordre de $10^{13}$ particules/s pendant environ $30 \mathrm{~min}$. Par contre, cette durée était beaucoup réduite pour les ions plus lourds, pour lesquels une intensité deux fois moindre entraînait la formation d'un trou correspondant à l'impact du faisceau en une dizaine de minutes. Cette durée était en général suffisante pour effectuer la mesure. Cependant, le dispositif a été modifié pour permettre des mesures répétées, par l'utilisation de cibles de forme allongée. que l'on peut déplacer par translation de l'extérieur de la chambre. On opère généralement ce déplacement avant que le formvar n'ait été altéré par le faisceau, ce qui garantit une cible d'épaisseur constante pendant toute la durée de la mesure.

2.3 LES FEUILLES D'ARRÊT. - La feuille destinée à arrêter le faisceau d'ions lourds pour protéger le détecteur était en or, ce qui convient très bien lorsque l'énergie du faisceau est inférieure à la barrière d'interaction correspondant à cet élément. Pour des énergies plus élevées, des feuilles de thorium ont également été utilisées. L'épaisseur de ces feuilles d'arrêt était choisie légèrement supérieure au parcours des ions lourds dans le matériau choisi (Au ou Th) [3].

2.4 LE DÉTECTEUR. - L'épaisseur du détecteur (jonction $\mathrm{Si}(\mathrm{Li})$ ) doit être suffisante pour arrêter totalement les protons de recul. L'énergie de ces protons est voisine de $4 E / A \mathrm{MeV}$ et atteint donc $24 \mathrm{MeV}$ pour des ions de $6 \mathrm{MeV} /$ nucléon, ce qui correspond à un parcours de $3,5 \mathrm{~mm}$ dans le silicium.

La surface du détecteur peut être faible, puisqu'un diaphragme de diamètre $3 \mathrm{~mm}$ est placé devant. Une grande surface risque d'augmenter le bruit de fond dû aux particules parasites (photons, neutrons) traversant la matière constituant le diaphragme.

Nous avons utilisé deux détecteurs, l'un de surface égale à $150 \mathrm{~mm}^{2}$ et d'épaisseur égale à $4,5 \mathrm{~mm}$, l'autre de surface égale à $50 \mathrm{~mm}^{2}$ et d'épaisseur égale à $5 \mathrm{~mm}$ (ce qui correspond au parcours des protons de $30 \mathrm{MeV}$ dans $\mathrm{Si}$ ). La résolution de ces deux détecteurs sur la raie à $8,785 \mathrm{MeV}$ de ${ }^{212}$ Po était de l'ordre de $60 \mathrm{keV}$.

L'étalonnage en énergie du détecteur était réalisé à l'aide des raies $\alpha$ d'un dépôt actif de thoron. La source était placée de façon à être vue de façon permanente par le détecteur, ce qui élimine les problèmes de dérive. L'énergie des principales raies $\alpha$ correspondant à cette source est égale à $6,051 \mathrm{MeV}(72 \%)$, $6,090 \mathrm{MeV}(28 \%)$ et $8,785 \mathrm{MeV}$. La résolution du détecteur ne permettait pas de séparer les deux premières de ces raies. Deux techniques différentes ont été utilisées pour résoudre ce problème :

- la méthode du centroïde consiste à calculer le centroïde de l'ensemble des deux raies voisines de $6 \mathrm{MeV}$ et de lui associer l'énergie

$$
E=0,72 \times 6,051+0,28 \times 6,090=6,062 \mathrm{MeV} .
$$

Le calcul du centroïde s'effectue par l'équation :

$$
G=\sum_{i=i_{0}}^{i_{1}} N_{i} C_{i} / \sum_{i=i_{0}}^{i_{1}} C_{i}
$$

$i_{0}$ et $i_{1}$ étant les canaux correspondant au début et à la fin du pic, $N_{i}$ et $C_{i}$ le numéro et le contenu du canal $i$; - la méthode de la médiane consiste à déterminer le canal $\mathrm{M}$ tel que la moitié des événements sont classés dans les canaux avant un numéro plus faible, c'est-àdire, pour un pic bien séparé, donné par la relation

$$
\sum_{i=i_{0}}^{\mathrm{M}} C_{i}=\frac{1}{2} \sum_{i=i_{0}}^{i_{1}} C_{i}
$$

avec les notations définies ci-dessus. La médiane correspondant à la raie à $6,051 \mathrm{MeV}$ était calculée par la relation

$$
\sum_{i=i_{0}}^{\mathrm{M}} C_{i}=0,36 \sum_{i=i_{0}}^{i_{1}^{\prime}} C_{i}
$$

la sommation (de $i_{0}$ à $i_{1}^{\prime}$ ) étant étendue à l'ensemble des deux raies à 6,051 et $6,090 \mathrm{MeV}$ (dont le pic à $6,051 \mathrm{MeV}$ représente $72 \%$ ). Ce procédé suppose que la montée de la première raie n'est pas perturbée par la traîne de la seconde. Pour notre résolution expérimentale de $50 \mathrm{keV}$ environ à $6 \mathrm{MeV}$, cette hypothèse entraîne une erreur inférieure à $1,5 \mathrm{keV}$ sur la position du pic, qui se traduit par une erreur relative inférieure à $0,025 \%$ sur la détermination de l'énergie du pic de protons diffusés (donc négligeable).

Nous avons d'ailleurs vérifié dans tous les cas que la méthode du centroïde et la méthode de la médiane aboutissaient au même étalonnage en énergie de notre système, à mieux que $\pm 0,5 \%$.

3. Précision des résultats. - Les sources d'incertitude sont les suivantes:

- La linéarité de la chaine électronique a été vérifiée à l'aide d'un générateur d'impulsions ORTFC, 
et cette mesure a montré que les éventuels défauts de linéarité ne pouvaient engendrer une erreur supérieure à $\pm 0,02 \%$.

- L'incertitude sur la pente de la droite d'étalonnage $\alpha$ est de l'ordre de $0,5 \%$. L'énergie du pic $\alpha$ à $8,785 \mathrm{MeV}$ étant bien déterminée, cette incertitude ne s'applique que sur' l'écart existant entre l'énergie du pic de proton et $8,785 \mathrm{MeV}$.

Pour des particules de $17 \mathrm{MeV}$ (protons correspondant à des faisceaux de 4,2 MeV/nucléon), l'incertitude relative résultante vaudra donc $0,25 \%$.

Cependant, la calibration ainsi obtenue pour des particules $\alpha$ n'est pas strictement valable pour des protons. K. W. Kemper et J. D. Fox ont en effet signalé [5] qu'il existe des différences de réponses d'un détecteur de silicium à barrière de surface à des protons et des particules $\alpha$ accélérés par un Tandem, à la même énergie. Ces auteurs ont observé que les particules $\alpha$ produisaient des impulsions plus élevées que les protons. Pour une même énergie de $9,5 \mathrm{MeV}$, l'écart était de $56 \pm 9 \mathrm{keV}$ soit $0,59 \%$. Il est clair que dans le cadre de l'application de la méthode présentée ici, où un détecteur étalonné à l'aide de particules $\alpha$ est utilisé pour mesurer l'énergie de protons diffusés, un tel effet conduit à sous-estimer l'énergie des protons donc du faisceau d'ions lourds. Nous avons, pour notre part, comparé [6] les réponses du détecteur de silicium pour des protons d'énergie comprise entre 12 et $24 \mathrm{MeV}$ aux énergies déduites de la droite d'étalonnage obtenue avec les particules $\alpha$. Les protons d'énergie variable et bien déterminée étaient obtenus par diffusion élastique sur une cible d'Au à partir d'un faisceau accéléré par le Tandem MP d'Orsay. Nous avons observé un effet identique à celui mentionné par Kemper et Fox. Pour une même énergie incidente les réponses du détecteur aux deux types de particules sont différentes. Dans tout le domaine d'énergie exploré, l'écart observé en énergie est de $0,68 \%$. On remarquera que cette valeur est très voisine de celle donnée par Kemper et Fox pour une énergie plus basse. Nous avons tenu compte de ces résultats en appliquant aux mesures d'énergie déduites de l'étalonnage pour les particules $\alpha$ une correction systématique de $+0,68 \%$ à laquelle est attachée une incertitude de $\pm 20 \%$.

- L'incertitude provenant de la perte d'énergie des particules dans la fenêtre d'entrée du détecteur est négligeable. En effet, cette fenêtre est égale à $40 \mu \mathrm{g} / \mathrm{cm}^{2}$, ce qui correspond à une perte d'énergie de l'ordre de $0,1 \mathrm{keV}$ pour les particules $\alpha$ de $6 \mathrm{MeV}$. .

- L'incertitude sur la position du pic dépend du nombre d'événements observés et du bruit de fond. Dans la plupart des cas, la méthode du centroïde, ou de la médiane permet, après soustraction du bruit de fond, de déterminer la position du pic à \pm deux canaux près, pour un pic situé vers le canal 2000. Cela correspond à une incertitude de l'ordre de $\pm 0,1 \%$.
- L'incertitude sur le ralentissement des protons dans la feuille d'arrêt des ions lourds est uniquement déterminée par l'incertitude sur les pouvoirs d'arrêt, l'épaisseur des feuilles étant bien connue. Ainsi qu'il a été dit plus haut, les tables de Williamson et Boujot [2] ont été utilisées pour déterminer les pertes d'énergie $\Delta E_{\mathrm{p}}$ des protons dans les feuilles d'arrêt. Nous avons pour trois énergies de protons 15,20 et $24 \mathrm{MeV}$, mesuré le ralentissement dans une feuille d'or de $41,97 \mathrm{mg} \cdot \mathrm{cm}^{-2}$. Le tableau I montre les résultats.

\section{TABLEAU I}

\begin{tabular}{|c|c|c|c|}
\hline $\begin{array}{l}\text { Energie des } \\
\text { protons }\end{array}$ & $\begin{array}{r}\Delta E_{\mathrm{p}} \\
\text { mesuré }\end{array}$ & $\begin{array}{l}\Delta E_{\mathrm{p}} \text { d'après } \\
\text { la Réf. [2] }\end{array}$ & Ecart \\
\hline - & - & - & . \\
\hline $15 \mathrm{MeV}$ & $587 \mathrm{keV} \pm 3 \%$ & $575 \mathrm{keV}$ & $+2 \%$ \\
\hline $20 \mathrm{MeV}$ & $473 \mathrm{keV} \pm 2,5 \%$ & $473 \mathrm{keV}$ & $0 \%$ \\
\hline $24 \mathrm{MeV}$ & $421 \mathrm{keV} \pm 2 \%$ & $416 \mathrm{keV}$ & $+1,2 \%$ \\
\hline
\end{tabular}

Compte tenu de cette confrontation, nous avons adopté, pour les pouvoirs d'arrêt, l'incertitude expérimentale de $\pm 3 \%$, ce qui correspond à une erreur de $\pm 0,06 \%$ sur l'énergie des protons.

- L'incertitude sur la perte d'énergie du faisceau incident dans le formvar est imposée par l'erreur sur le pouvoir d'arrêt de ces feuilles pour les ions considérés. Nous avons adopté une valeur de $\pm 20 \%$ (comprenant l'incertitude de $\pm 2 \%$ sur l'épaisseur des feuilles) ce qui ajoute une erreur de $\pm 0,1 \%$ sur l'énergie incidente.

- Enfin, il faut tenir compte de l'incertitude existant sur l'angle de détection des protons.

Nous avons vu que les diaphragmes utilisés définissent une ouverture angulaire de $\pm 3^{\circ}$ autour de l'axe $\mathrm{du}$ système. Cela correspond à une valeur maximale de l'angle de diffusion $\theta_{\max }$ égale à $3^{\circ}$ dans l'hypothèse d'un alignement parfait du système par rapport au faisceau, et d'une convergence initiale nulle pour celui-ci. Compte tenu de la non-réalisation de ces conditions idéales (l'alignement du système au laser ou à la lunette pouvant entraîner une erreur de l'ordre du degré, et la convergence du faisceau étant du même ordre de grandeur), il est raisonnable de supposer que certains protons observés correspondent à un angle de diffusion de 4 ou 5 degrés. Le calcul dans le système du laboratoire de la distribution angulaire des protons diffusés élastiquement (diffusion de Rutherford) montre d'autre part que la section efficace différentielle $(\mathrm{d} \sigma / \mathrm{d} \Omega)$ est à peu près indépendante de l'angle $\theta$ dans le domaine $0-5^{\circ}$, pour les projectiles étudiés. Par conséquent, le nombre de protons diffusés, proportionnel à $\sin \theta(\mathrm{d} \sigma / \mathrm{d} \Omega)$ croît linéairement avec l'angle dans ce domaine angulaire.

Dans ces conditions, nous avons supposé que le maximum de la distribution en énergie des protons observés correspond à un angle moyen $\bar{\theta}=3_{-3}^{+1,5}$. L'énergie mesurée est alors trop faible d'un facteur égal à $\cos ^{2} \bar{\theta}=0,997 \pm 0,003$. 
Nous avons donc appliqué à l'énergie des protons une correction systématique d'un facteur égal à $1,003 \pm 0,003$.

La somme quadratique des erreurs (regroupées dans le tableau II) conduit à une incertitude relative de l'ordre de $\pm 0,45 \%$.

\section{TABLEAU II}

Correction pour les protons

Position pic

Ralentissement protons

Perte d'énergie dans formvar

Angle de détection

Erreur résultante
Source d'erreur

Pente droite d'étalonnage $\alpha$

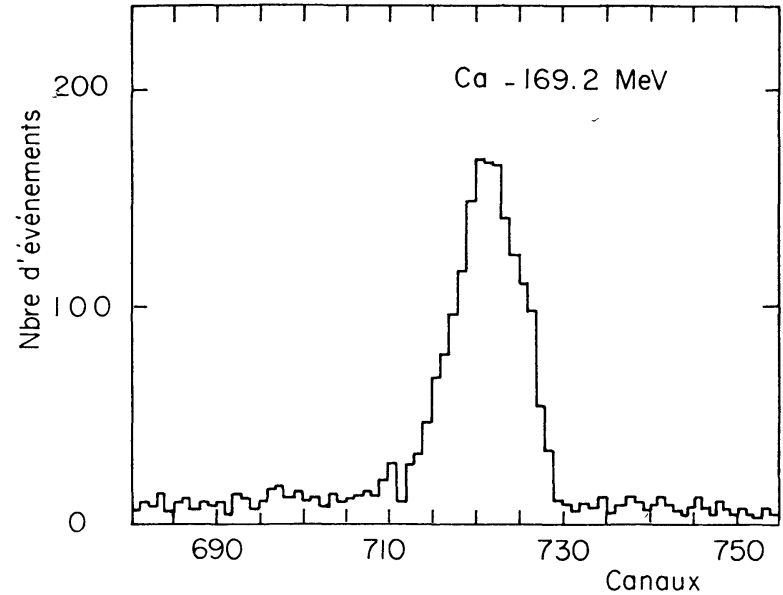

FIG. 3. - Spectre obtenu pour ${ }^{40} \mathrm{Ca}$ à $169,2 \mathrm{MeV}$.

[Proton spectrum obtained with $169.2 \mathrm{MeV}{ }^{40} \mathrm{Ca}$ ions.]

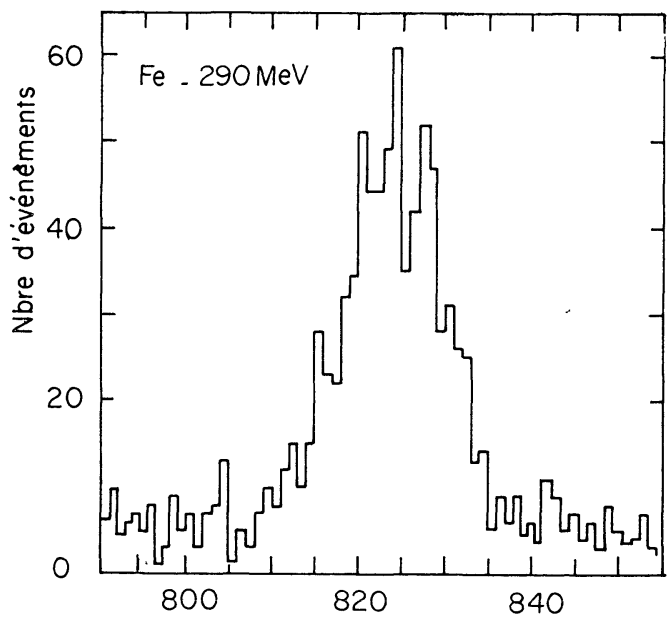

FIG. 4. - Spectre obtenu pour ${ }^{56} \mathrm{Fe}$ à $290 \mathrm{MeV}$.

[Proton spectrum obtained with $290 \mathrm{MeV}{ }^{56} \mathrm{Fe}$ ions.]

sur des faisceaux de fluor et de calcium accélérés par le Tandem MP. L'énergie était connue avec une précision de l'ordre de $10^{-4}$ par une mesure magnétique utilisant un aimant de déviation étalonné avec précision. Les résultats de ces mesures sont donnés dans le tableau III où on peut constater une très bonne détermination de l'énergie des ions lourds par notre méthode. L'écart relatif entre la valeur mesurée par notre dispositif $\left(E_{2}\right)$ et la valeur donnée par la méthode

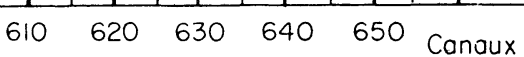

FIG. 2. - Spectre obtenu pour ${ }^{19} \mathrm{~F}$ à $80 \mathrm{MeV}$.

[Proton spectrum obtained with $80 \mathrm{MeV}{ }^{19} \mathrm{~F}$ ions.]

4.2 Contrôle Des VAlEurs ABsolues SUR DES FAISCEAUX DU TANDEM. - La précision sur la valeur absolue déterminée par cette mesure a été contrôlée

\section{TABLEAU III}

\begin{tabular}{|c|c|c|c|}
\hline & & LEAU III & \\
\hline & $\begin{array}{l}\text { Energie } \\
\text { (aimant) }\end{array}$ & $\begin{array}{l}\text { Energie } \\
\text { mesurée }\end{array}$ & $\begin{array}{c}\text { Ecart relatif } \\
\text { observé }\end{array}$ \\
\hline Ion & $E_{1}(\mathrm{MeV})$ & $E_{2}(\mathrm{MeV})$ & $\left(E_{2}-E_{1}\right) / E_{1}$ \\
\hline- & - & - & -1 \\
\hline${ }^{19} \mathrm{~F}$ & 80,00 & 80,18 & $+0,23 \%$ \\
\hline${ }^{40} \mathrm{Ca}$ & 142,15 & 142,5 & $+0,25 \%$ \\
\hline & 156,00 & 156,0 & $0 \%$ \\
\hline & 169,20 & 169,8 & $+0,35 \%$ \\
\hline
\end{tabular}


de déviation magnétique $\left(E_{1}\right)$ est de l'ordre de $0,3 \%$, c'est-à-dire inférieure à l'incertitude calculée de $\pm 0,45 \%$.

5. Conclusion. - Parmi les caractéristiques du dispositif qui vient d'être décrit, il convient de noter particulièrement sa simplicité et sa facilité de mise en œuvre. Son encombrement réduit (sa longueur est inférieure à $20 \mathrm{~cm}$ ) permet de le monter sans difficulté sur les installations qui équipent généralement les lignes de faisceau (chambres à réactions, boîtes de visualisation, etc...). Ces qualités ne sont pas obtenues au détriment de la précision puisque celle-ci est de $\pm 0,45 \%$ pour des énergies d'ions lourds allant jusqu'à $6 \mathrm{MeV} /$ nucléon. Au-delà les sections efficaces de diffusion élastique ions lourds/protons diminuent très fortement, et les spectres de protons projetés se superposent à un bruit de fond rapidement croissant. Les mesures restent possibles, mais sont longues et moins précises.

Ce dispositif permet de contrôler ou de calibrer des installations, plus complexes du point de vue mécanique ou électronique, utilisables pour la détermination de l'énergie des ions lourds : déviation magnétique, temps de vol. Dans ce sens, une étude est en cours auprès de l'accélérateur ALICE d'Orsay [7] où l'aimant d'aiguillage du faisceau dans la salle d'expériences est utilisé dans une direction donnée pour des mesures d'énergie (détermination du champ magnétique de déviation du faisceau à l'aide d'une sonde $\mathrm{RMN}$ ). Ce dispositif a aussi été récemment utilisé [8] auprès de l'accélérateur UNILAC à Darmstadt (R.F.A.) pour contrôler les mesures d'énergie du faisceau par temps de vol des ions.

Enfin le dispositif est conçu pour permettre le positionnement d'absorbeurs solides entre les diaphragmes $D_{0}$ et $D_{1}$ (voir Fig. 1) et rendre ainsi possible la mesure des pertes d'énergie dans ces matériaux par différence entre l'énergie absolue sans et avec ralentisseur. Il a ainsi été utilisé pour des mesures de pouvoirs d'arrêt d'ions ${ }^{16} \mathrm{O},{ }^{40} \mathrm{Ar},{ }^{63} \mathrm{Cu}$ et ${ }^{84} \mathrm{Kr}$ dans des ralentisseurs de $\mathrm{C}, \mathrm{Al}, \mathrm{Ni}, \mathrm{Ag}$ et $\mathrm{Au}$ [4].

Remerciements. - Nous tenons à remercier les équipes de conduite des accélérateurs ALICE et TANDEM de l'I.P.N. d'Orsay pour leur collaboration efficace.

\section{Bibliographie}

[1] Olsen, D. K., Merrill, N. M., Biagi, S. F., Phillips, N. R. BARNETT, A. R., Nucl. Instrum. Methods 114 (1974) 615

[2] Williamson, C. F., Boujot, J. P., Picard, J., Rapport CEA R-3042 (1966).

[3] Northcliffe, L. C., Schilling, R. F., Nucl. Data Tables A 7 (1970) 233.

[4] Bimbot, R., Della Negra, S., Gardès, D., Gauvin, H. Fleury, A., Hubert, F., IPNO-RC-77-11 et $\mathrm{Nucl}$. Instrum. Methods (à paraître).
[5] Kemper, K. W. et Fox, J. D., Nucl. Instrum. Methods 105 (1972) 333.

[6] Bimbot, R., Cabot, C., Gardès, D., Gauvin, H., Rivet, M. F. (soumis à Nucl. Instrum. Methods).

[7] Bimbot, R., Cabot, C., Depauw, J., Gardès, D., Gauvin, H., Martial, L., Richomme, A., Rivet, M. F. (à paraître). [8] Вimbot, R., Gardès, D., Geissel, H. et Schmidt, K.H.Z. Phys. A 286 (1978) 327. 